\title{
Clinical significance of FBXW7 tumor suppressor gene mutations and expression in human colorectal cancer: a systemic review and meta-analysis
}

Wei Shang ${ }^{1 \dagger}$, Chuanwang Yann ${ }^{2 \dagger}$, Ran $\mathrm{Liu}^{1 \dagger}$, Lili Chen ${ }^{3}$, Dongdong Cheng ${ }^{4}, \mathrm{Liang} \mathrm{Hao}^{5}$, Wenguang Yuan ${ }^{1}$, Jingbo Chen ${ }^{1 *}$ and Hui Yang ${ }^{1 *}$ (D)

\begin{abstract}
Background: Various studies investigating the clinical significance of FBXW7 mutation and/or expression have yielded inconclusive results in colorectal cancer (CRC) patients. Therefore, the present meta-analysis summarizes previous evidence and evaluates the clinical significance, including the prognostic role, of FBXW7 status in CRCs.

Methods: The meta-analysis was conducted by searching the databases of PubMed, China National Knowledge Infrastructure (CNKI), WANFANG data, Web of Science, Embase, and Web of Science. Pooled odds ratios (ORs) and hazard ratios (HRs) and corresponding 95\% confidence intervals ( $\mathrm{Cls}$ ) were calculated to assess the relationships between FBXW7 status and clinicopathological features and survival in CRC, respectively.

Results: Ten studies involving 4199 patients met the inclusion criteria and included in our meta-analysis. FBXW7 mutation/low expression was obviously correlated with advanced T stage ( $\mathrm{OR}=0.44,95 \% \mathrm{Cl}: 0.27-0.74, P<0.01)$ and lymph node metastasis $(\mathrm{OR}=1.88,95 \% \mathrm{Cl}: 1.40-2.53, P<0.01)$, but was not associated with other parameters. Further investigation found that FBXW7 mutation/low expression predicted poor OS ( $\mathrm{HR}=1.25,95 \% \mathrm{Cl}$ : 1.06-1.47, $P<0.01)$, but not DFS in CRC (HR=1.04, 95\% Cl: $0.60-1.82, P=0.88)$. Subgroup analysis found that FBXW7 status was obviously correlated with OS in cohorts recruited after 2009 (HR=1.32, 95\% Cl: 1.17-1.50, $P<0.01$ ), from eastern Asia ( $H R=1.27,95 \% \mathrm{Cl}: 1.04-1.55, P=0.02)$, detected by immunohistochemistry/qRT-PCR (HR $=1.39,95 \% \mathrm{Cl}$ : $1.22-1.59, P<0.01)$, and analysed with multivariate method ( $H R=1.47,95 \% \mathrm{Cl}: 1.25-1.74, P<0.01)$.

Conclusions: This study indicates that FBXW7 status, expression level especially, is associated with OS but not DFS in CRC. FBXW7 expression level may function as a prognostic biomarker in CRC.
\end{abstract}

Keywords: FBXW7, Mutation, Expression, Survival, Cancer

\footnotetext{
*Correspondence: qychenjingbo@163.com; yanghgfshospital@163.com

†Shang Wei, Yan Chuanwang and Liu Ran contributed equally to this work.

'Department of General Surgery, Key Laboratory of Metabolism and Gastrointestinal Tumor, Key Laboratory of Laparoscopic Technology, Shandong Medicine and Health Key Laboratory of General Surgery, The First Affiliated Hospital of Shandong First Medical University \& Shandong Provincial Qianfoshan Hospital, the First Affiliated Hospital of Shandong First Medical University, Jinan 250000, Shandong, China

Full list of author information is available at the end of the article
}

(c) The Author(s). 2021 Open Access This article is licensed under a Creative Commons Attribution 4.0 International License, which permits use, sharing, adaptation, distribution and reproduction in any medium or format, as long as you give appropriate credit to the original author(s) and the source, provide a link to the Creative Commons licence, and indicate if changes were made. The images or other third party material in this article are included in the article's Creative Commons licence, unless indicated otherwise in a credit line to the material. If material is not included in the article's Creative Commons licence and your intended use is not permitted by statutory regulation or exceeds the permitted use, you will need to obtain permission directly from the copyright holder. To view a copy of this licence, visit http://creativecommons.org/licenses/by/4.0/ The Creative Commons Public Domain Dedication waiver (http://creativecommons.org/publicdomain/zero/1.0/) applies to the data made available in this article, unless otherwise stated in a credit line to the data. 


\section{Background}

Colorectal cancer (CRC) ranks the forth most commonly diagnosed cancer and the second leading cause of cancerrelated death worldwide [1]. Based on the most recent data, the annual age standardized CRC incidence rate was 38.7 per 100,000 persons (2012-2016), and the mortality rate was 13.9 per 100,000 persons (2013-2017) [2]. Despite recent advances in therapy and multidisciplinary care in CRC, about 900,000 individuals die from this malignancy [3]. Fortunately, recent advances in genomic sequencing and molecular based cancer development pathways now allow for a deeper understanding of pathogenesis [4]. Some well-known genes in CRC may provide opportunities for targeted clinical interventions or/and survival prediction.

FBWX7 (F-box and WD repeat domain-containing 7) is the substrate recognition component of an evolutionary conserved SCF (complex of SKP1, CUL1 and F-box protein)-type ubiquitin ligase [5]. Functioning as a general tumor suppressor in human cancer, FBXW7 is the most frequently mutated of SCF-type ubiquitin ligase in human cancer cells [6]. Besides, it has been shown to degrade several proto-oncogenes that function in cellular growth and division pathways, including cyclin E1, cMyc, c-Jun, and Notch [7]. The altered status of FBXW7 is recognized to be one of the major causes of carcinogenesis or cancer development $[5,7,8]$. CRC harbors the second most frequent FBXW7 mutations (7.73\%) among different cancer types [9]. Moreover, FBXW7 is one of the most frequently mutated genes during CRC initiation and progression [10]. Altered FBXW7 status (mutation and/or low expression) may be associated with prognosis in CRC, however, the results vary among different studies [11-20]. Thus, we conducted a systematic review and meta-analysis of data from previous studies to quantitatively assess the association between FBXW7 status and survival in CRC.

\section{Methods}

\section{Literature search and study selection}

A systematic literature search of PubMed, China National Knowledge Infrastructure (CNKI), WANFANG data, Web of Science, Embase, and Web of Science was performed in September, 2020. The following key words or text words were used: "FBXW7", "CDC4", "CRC", "colon", "rectum", "intestinal", "cancer", "carcinoma", "tumor", "prognosis", "survival". Eligible articles should meet the following criteria: (1) CRC was pathologically confirmed; (2) studies investigated the association of FBXW7 mutation and/or expression with survival outcome; (3) the hazard ratio (HR) and 95\% confidence interval (CI) for survival were provided or could be calculated from the available data. Articles were excluded based on any of the following criteria: (1) studies lacking essential information for calculating HR and 95\% CI; (2) reviews, comments, letters, case reports, and conference abstracts; (3) neither English nor Chinese articles. When multiple publications of a study were identified, the most detailed version for meta-analysis was selected. A flow diagram of the study selection process is presented in Fig. 1.

\section{Data extraction}

Two reviewers (WS and CWY) independently extracted the following data from each study: basic study information (name of first author, year of publication, region or country where the study was conducted, number of patients, follow-up period, and analysis method of survival), participant characteristics (age and gender), FBXW7 related data (detection method, cutoff score, antibody source and dilution, the HRs of FBXW7 mutation/expression for overall survival (OS), disease-free survival (DFS), as well as their 95\% CIs and $P$ values) and clinical parameters (histological type, tumor size,

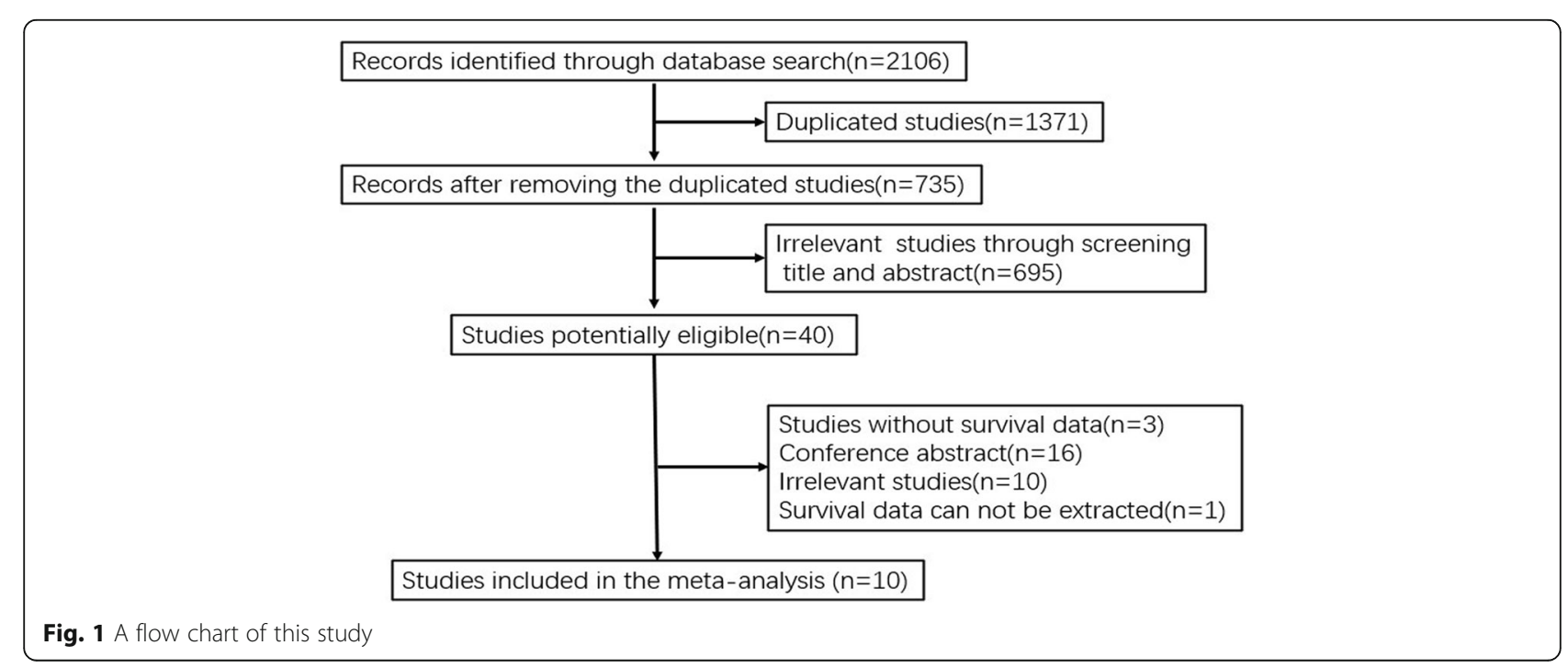


tumor location, venous invasion, peritoneal metastasis, lymph node metastasis, distant metastasis, TNM stage and Duke's stage). If available, HRs and 95\% CIs were preferentially obtained from multivariate results. Otherwise, they were extracted from univariable outcomes or calculated using Engauge Digitizer version 4.1 (free software down-loaded from http://sourceforge.net) to read the Kaplane-Meier survival curves to get the HRs and 95\% CIs [21-23]. Discrepancies were adjudicated by a third reviewer (RL) until a consensus was reached.

\section{Quality assessment}

The quality of all eligible studies were assessed independently by 2 investigators (CWY and RL) using the Newcastle-Ottawa quality assessment scale (NOS). All disagreements were discussed and resolved with consensus. The NOS criteria was scored based on three aspects: (1) subject selection, (2) comparability of subject, (3) clinical outcome. Scores based on NOS of 7-9 indicate a goodquality study, scores of 4-6 indicate an intermediate-quality study, and scores less than 4 indicate a low-quality study.

\section{Statistical analysis}

Statistical analysis was performed using Stata statistical software version 12.0 (Stata Corporation, College Station, Texas, USA) and Review Manager version 5 (RevMan; The Nordic Cochrane Centre, Copenhagen, Denmark). Odds ratios (ORs) with 95\% confidence intervals (CIs) were estimated to evaluate the association between FXBW7 status and the clinicopathological features in CRC. The statistical significance of the pooled OR and HR was evaluated with the $\mathrm{Z}$ test and $P$ values, and $P<0.05$ was considered statistically significant. Subgroup analysis was conducted to determine the source of existing heterogeneity. Heterogeneity among studies was determined by employing the $\mathrm{Q}$ and $\mathrm{I}^{2}$ statistics. If the value was greater than 0.1 and the ${ }^{2}$ value was less than $50 \%$, the heterogeneity among studies did not reach statistical significance, and the fixed-effects model was subsequently implemented. Otherwise, the random-effects model was used. Publication bias was assessed by the Begg's rank correlation method and Egger's weighted regression method, and a $P$ value less than 0.05 was considered statistically significant. In addition, a sensitivity analysis was performed to assess the influence of a single study on pooled HR.

\section{Results}

\section{Study selection and description of the include studies}

A total of 2106 articles were obtained through database search. After removing duplicated studies and irrelevant studies through screening title and abstract, 40 studies were remained. Then, the full texts of the articles were reviewed in detail, and 10 studies met our inclusion criteria were finally included for the meta-analysis, including
4 studies detecting FBXW7 mutation and 6 studies measuring FBXW7 expression. The main characteristics of the included studies are presented in Table 1 . These studies were published between 2009 and 2019, and conducted in 4 countries (China, Australia, America, and Japan). The overall sample size was 4199, ranging from 50 to 1519 . The relationship between OS and FXBW7 status was all described in the 10 studies, and DFS was reported in 4 studies. All of the eligible entries scored more than five by NOS, revealing a high methodological quality across all studies. FBXW7 expression was measured by IHC or qRT-PCR, and mutation was detected though different sequencing methods. For the purposes of this anlaysis, cases with low expression of FBXW7 or coding mutations were considered one similar group of patients that had tumors with a deficit in FBXW7.

\section{Correlation between FBXW7 and clinicopathological features}

Correlation between FBXW7 status and clinicopathological features was presented in 8 studies. Based on the ORs derived from these studies, we evaluated the correlation between FBXW7 status and some clinicopathological characteristics, including age, gender, histological grade, tumor size, tumor location, venous invasion, peritoneal metastasis, depth of invasion, lymph node metastasis, distant metastasis, TNM stage and Duke's stage. (Table 2) Aberrant FBXW7 status was significantly associated with advanced $\mathrm{T}$ stage $(\mathrm{OR}=0.44,95 \% \mathrm{CI}$ : $0.27-$ $0.74, P<0.01)$ and lymph node metastasis $(\mathrm{OR}=1.88$, 95\% CI: $1.40-2.53, P<0.01)$. Frequency of venous invasion was also higher in FBXW7 mutation/low expression cohort, but no statistical significance was detected $(\mathrm{OR}=$ 1.63, 95\% CI: 1.01-2.64, $P=0.05$ ). No obvious relationship was verified between FBXW7 status and other parameters. (Table 2).

\section{Prognostic value of FBXW7}

All the 10 studies were enrolled to detect the prognostic value of FBXW7 in OS. A random-effect model was used to calculate the pooled HR and 95\% CI because excessive heterogeneity existed between studies $\left(P<0.01, \mathrm{I}^{2}=73 \%\right)$. (Fig. 2a) Overall, FBXW7 mutation/low expression predicted poor OS (HR $=1.25,95 \%$ CI: $1.06-1.47, P<0.01)$. (Fig. 2a) However, no significant correlation was found between FBXW7 and DFS in CRC (HR $=1.04$, 95\% CI: 0.60$1.82, P=0.88$ ). (Fig. $2 \mathrm{~b}$ ) To detect potential heterogeneity, subgroup analyses were stratified based on recruitment time, region, FBXW7 detection method, sample size and data type to evaluate FXBW7 prognostic value in CRC. As shown in Table 3, FBXW7 mutation/low expression predicted decreased OS regardless of sample size $\geq 100$ (HR = 1.23, 95\% CI: $1.01-1.51, P=0.04)$ or $<100(\mathrm{HR}=1.33$, 95\% CI: 1.09-1.63, $P<0.01)$. Besides, FBXW7 status was 


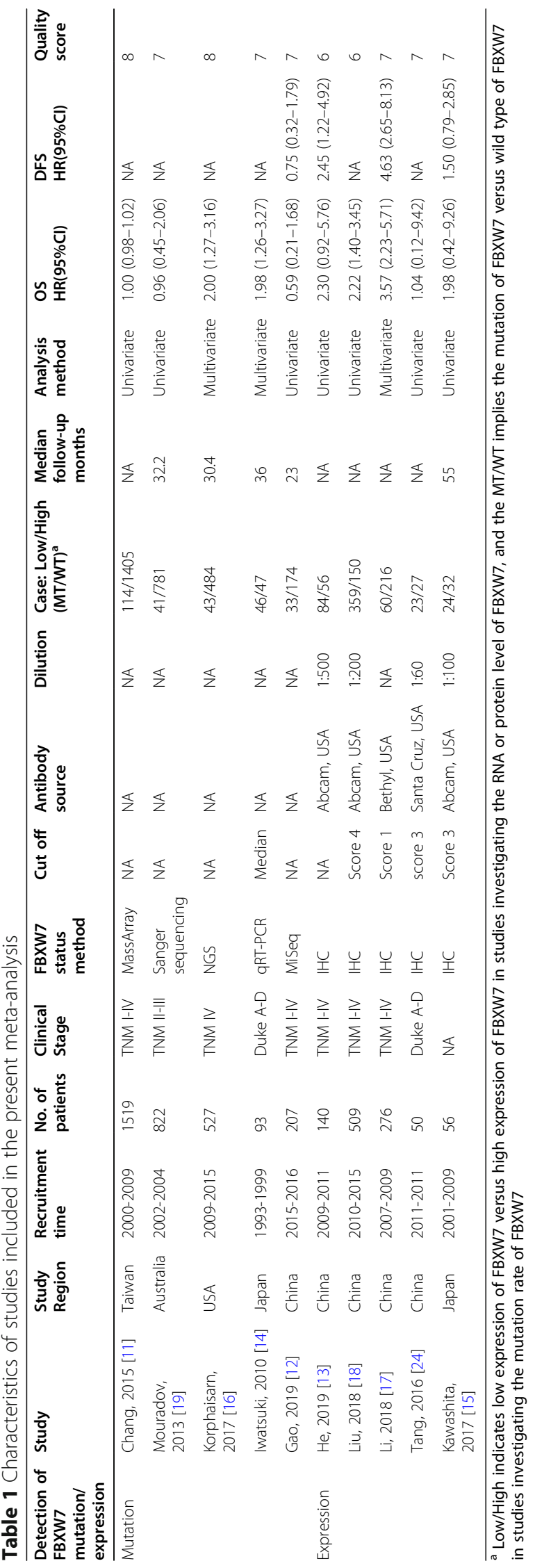


Table 2 Meta-analysis of FXBW7 status and clinicopathological features in CRC

\begin{tabular}{|c|c|c|c|c|c|c|}
\hline Parameters Characteristics & Number of studies & OR $(95 \% \mathrm{Cl})$ & $I^{2}(\%)$ & $P_{\mathrm{h}}$ & Z & $P$ value \\
\hline Age( $\geq 60$ year vs. $<60$ year $)$ & 3 & $1.00(0.93-1.36)$ & 0 & 0.71 & 0.00 & 1.00 \\
\hline Gender (Male vs. Female) & 7 & $1.03(0.83-1.28)$ & 6 & 0.38 & 0.28 & 0.78 \\
\hline Differentiation(Well vs. Moderate + Poor) & 2 & $0.81(0.40-1.64)$ & 0 & 0.63 & 0.89 & 0.37 \\
\hline Differentiation(Well+ Moderate vs. Poor) & 4 & $0.72(0.35-1.48)$ & 69 & 0.02 & 0.59 & 0.55 \\
\hline Size $(\geq 5 \mathrm{~cm}$ vs. $<5 \mathrm{~cm})$ & 3 & $0.93(0.64-1.35)$ & 0 & 0.45 & 0.37 & 0.71 \\
\hline Tumor location(Colon vs. Rectum) & 5 & $0.85(0.64-1.12)$ & 30 & 0.22 & 1.17 & 0.24 \\
\hline Venous invasion(Present vs. Absent) & 3 & $1.63(1.01-2.64)$ & 14 & 0.31 & 1.99 & 0.05 \\
\hline Peritoneal metastasis (Present vs. Absent) & 2 & $0.82(0.38-1.80)$ & 0 & 0.40 & 0.49 & 0.63 \\
\hline Depth of invasion (T1 + T2 vs. T3 + T4) & 3 & $0.44(0.27-0.74)$ & 0 & 0.99 & 3.12 & $<0.01$ \\
\hline Lymph node metastasis (Positive vs. Negative) & 5 & $1.88(1.40-2.53)$ & 0 & 0.45 & 4.18 & $<0.01$ \\
\hline Distant metastasis (Present vs. Absent) & 3 & $1.85(0.34-10.24)$ & 92 & $<0.01$ & 0.71 & 0.48 \\
\hline TNM stage( + + II vs. III + IV) & 3 & $0.53(0.15-1.84)$ & 95 & $<0.01$ & 1.00 & 0.32 \\
\hline Duke's stage $(A+B$ vs. $C+D)$ & 2 & $0.45(0.04-5.20)$ & 90 & $<0.01$ & 0.64 & 0.52 \\
\hline
\end{tabular}

obviously correlated with OS in cohorts recruited after $2009(\mathrm{HR}=1.32$, 95\% CI: $1.17-1.50, P<0.01)$, from eastern Asia $(\mathrm{HR}=1.27,95 \% \mathrm{CI}: 1.04-1.55, P=0.02)$, detected by $\mathrm{IHC} / \mathrm{qRT}-\mathrm{PCR} \quad(\mathrm{HR}=1.39,95 \% \quad \mathrm{CI}: 1.22-1.59$, $P<0.01)$, and analysed with multivariate method $(\mathrm{HR}=$ 1.47, 95\% CI: 1.25-1.74, $P<0.01)$. However, no prognostic effect was observed in patients recruited before $2009(\mathrm{HR}=1.24,95 \% \mathrm{CI}: 0.93-1.65, P=0.14)$, from regions beyond eastern Asia ( $\mathrm{HR}=1.18,95 \% \mathrm{CI}$ : 0.87-1.61, $P=0.28)$, detected by sequencing $(\mathrm{HR}=1.17,95 \% \mathrm{CI}$ : $0.94-1.47, P=0.16)$, and analysed with univariate method $(\mathrm{HR}=1.13$, 95\% CI: 0.94-1.35, $P=0.20)$. (Table 3).

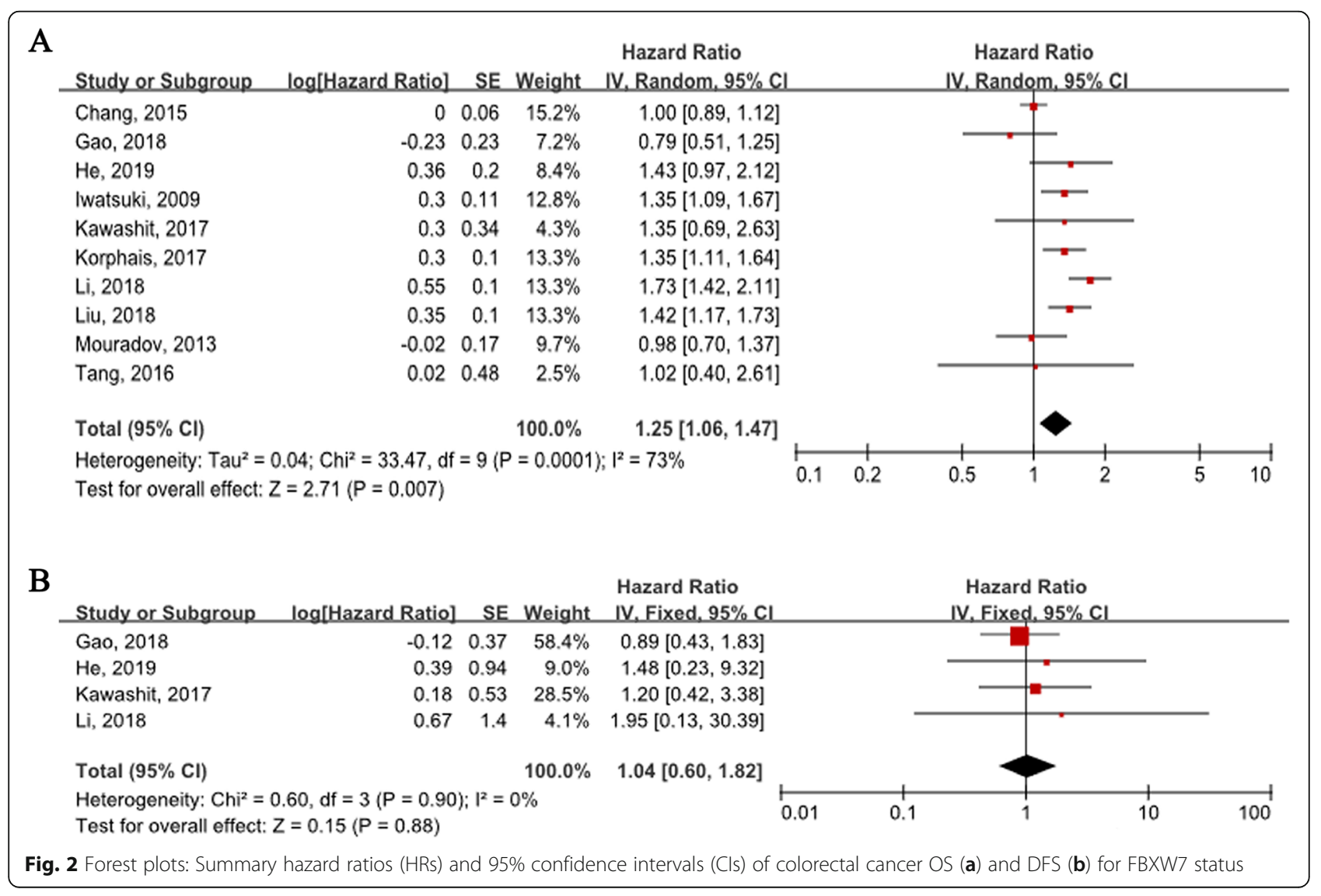


Table 3 Subgroup analyses for overall survival

\begin{tabular}{|c|c|c|c|c|c|c|c|}
\hline Outcome & Characteristics & Number of studies & $\mathrm{HR}(95 \% \mathrm{Cl})$ & $I^{2}(\%)$ & $P_{h}$ & Z & $P$ value \\
\hline \multirow[t]{2}{*}{ Recruitment time } & Before 2009 & 5 & $1.24(0.93-1.65)$ & 88 & $<0.01$ & 1.46 & 0.14 \\
\hline & After 2009 & 5 & $1.32(1.17-1.50)$ & 32 & 0.21 & 4.43 & $<0.01$ \\
\hline \multirow[t]{2}{*}{ Region } & Eastern asia & 8 & $1.27(1.04-1.55)$ & 77 & $<0.01$ & 2.31 & 0.02 \\
\hline & Other regions & 2 & $1.18(0.87-1.61)$ & 62 & 0.10 & 1.08 & 0.28 \\
\hline \multirow[t]{2}{*}{ FBXW7 detection method } & IHC/qRT-PCR & 6 & $1.39(1.22-1.59)$ & 46 & 0.10 & 4.95 & $<0.01$ \\
\hline & Sequencing & 4 & $1.17(0.94-1.47)$ & 73 & 0.01 & 1.40 & 0.16 \\
\hline \multirow[t]{2}{*}{ Sample Size } & $\geq 100$ & 7 & $1.23(1.01-1.51)$ & 81 & $<0.01$ & 2.06 & 0.04 \\
\hline & $<100$ & 3 & $1.33(1.09-1.63)$ & 0 & 0.85 & 2.81 & $<0.01$ \\
\hline \multirow[t]{2}{*}{ Data types } & Univariate & 7 & $1.13(0.94-1.35)$ & 56 & 0.03 & 1.28 & 0.20 \\
\hline & Multivariate & 3 & $1.47(1.25-1.74)$ & 50 & 0.13 & 4.56 & $<0.01$ \\
\hline
\end{tabular}

\section{Publication bias and sensitivity analysis}

A funnel plot, with regard to the publication bias of all studies for OS and four studies for DFS, showed the basic symmetrical. (Fig. 3a and b) Evaluation of publication bias using Begg's and Egger's tests also showed that no publication bias existed ( $P$ value of Begg's test, 0.24 and 0.31 for OS and DFS, respectively; $P$ value of Egger's test, 0.75 and 0.08 for OS and DFS, respectively). Furthermore, to evaluate the results of meta-analysis,

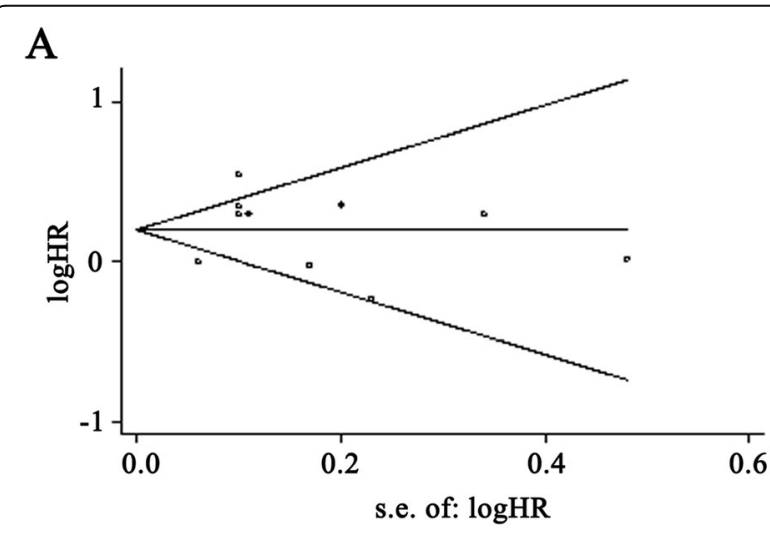

B

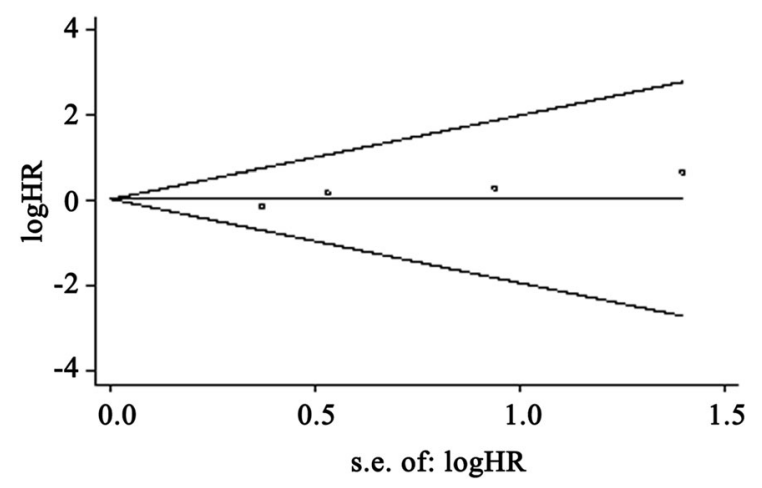

Fig. 3 Begg's funnel plots of the natural logarithm of the hazard ratios (HRs) and the SE of the natural logarithm of the HRs for the included studies reported with OS (a) and PFS (b) sensitivity analysis was conducted. No significant change was found in the results when any 1 study was excluded, confirming the robustness and reliability of metaanalysis results on both OS and DFS (Table 4).

\section{Discussion}

Our team has focused on investigating the functional role of FBXW7 in multiple cancers, including in CRC [25-28]. FBXW7 is one of most frequently mutated and downregualted genes in CRC, however, the clinical significance and prognostic value of FBXW7 in CRC have not been specified. To our known, this is the first meta-analysis to provide comprehensive evidence of the association between FBXW7 status and prognosis in CRC. Mutation in this study indicated all mutations whether accompanied with loss of function or not. Mutation detection has some advantages, for example, the mutation of FBXW7 can be detected in patients not underwent operation and cancer tissues can not be achieved, which are necessary for protein detecting. Pooled data of 4199 CRC patients confirmed that FBXW7 mutation and expression loss were detected in 7.5 and $53.0 \%$ cases, respectively. Previous study has indicated that FBXW7 could repress the migratory and invasive capacities of CRC cells through inhibiting stem cell-like behavior and epithelial-mesenchymal transition [26]. This meta-analysis suggests that FBXW7 mutation and/or low expression was significantly associated with advanced $\mathrm{T}$ stage and lymph node metastasis. Venous invasion rate was also higher in FBXW7 mutation/low expression cohort, though not statistically significant. This evidence indicates the essential role of FBXW7 in local invasion and metastasis in CRC. In addition, FBXW7 missense mutations have been shown to have a strong negative prognostic association in CRC [16], the association between FBXW7 status and distant metastasis was not discovered in our meta-analysis (OR $=1.85,95 \%$ CI: $0.34-10.24, P=0.48)$. Moreover, tumor size and clinical stage were not correlated with FBXW7 status as revealed in this study. Taken together, FBXW7 may 
Table 4 The influence of individual study on the pooled estimate for outcomes

\begin{tabular}{|c|c|c|c|c|c|c|}
\hline Outcome & Study omitted & $\mathrm{HR}(95 \% \mathrm{Cl})$ & $I^{2}(\%)$ & $P_{\mathrm{h}}$ & $\mathbf{Z}$ & $P$ value \\
\hline \multirow[t]{10}{*}{ OS } & Chang, 2015 [11] & $1.32(1.15-1.52)$ & 49 & 0.05 & 3.93 & $<0.01$ \\
\hline & $\mathrm{He}, 2019$ [13] & $1.23(1.04-1.47)$ & 76 & $<0.01$ & 2.38 & 0.02 \\
\hline & Mouradov, 2013 [19] & $1.28(1.08-1.52)$ & 75 & $<0.01$ & 2.83 & $<0.01$ \\
\hline & Liu, 2018 [18] & $1.22(1.02-1.47)$ & 74 & $<0.01$ & 2.18 & 0.03 \\
\hline & Korphaisarn, 2017 [16] & $1.23(1.02-1.48)$ & 75 & $<0.01$ & 2.21 & 0.03 \\
\hline & Iwatsuki, 2010 [14] & $1.19(1.03-1.38)$ & 59 & 0.01 & 2.39 & 0.02 \\
\hline & Li, 2018 [17] & $1.23(1.03-1.48)$ & 75 & $<0.01$ & 2.24 & 0.03 \\
\hline & Tang, 2016 [24] & $1.26(1.06-1.48)$ & 76 & $<0.01$ & 2.69 & $<0.01$ \\
\hline & Gao, 2019 [12] & $1.29(1.10-1.52)$ & 73 & $<0.01$ & 3.10 & $<0.01$ \\
\hline & Kawashita, 2017 [15] & $1.24(1.05-1.47)$ & 76 & $<0.01$ & 2.55 & 0.01 \\
\hline \multirow[t]{4}{*}{ DFS } & $\mathrm{He}, 2019$ [13] & $1.01(0.56-1.80)$ & 0 & 0.80 & 0.03 & 0.97 \\
\hline & Li, 2018 [17] & $1.02(0.58-1.79)$ & 0 & 0.82 & 0.06 & 0.95 \\
\hline & Gao, 2018 [12] & $1.31(0.56-3.11)$ & 0 & 0.94 & 0.62 & 0.53 \\
\hline & Kawashita, 2017 [15] & $0.99(0.51-1.91)$ & 0 & 0.78 & 0.03 & 0.98 \\
\hline
\end{tabular}

influence the survival outcomes of CRC patients through regulating local invasion and lymph node metastasis but not tumor growth.

Our meta-analysis found that FBXW7 mutation/low expression predicted poor OS, but not DFS in CRC. When subgroup analysis was conducted, FBXW7 status was correlated with OS in cohorts analysis with multivariate method, but not with univariate method. Results from multivariate analysis, which took other clinicopathological parameters into consideration simultaneously, are more accurate than univariate analysis. Besides, FBXW7 mRNA/protein level was correlated with OS, but FBXW7 mutation alone was not. Previous study has found that FBXW7 mutations are not predicted to cause loss of function [29]. FBXW7 mRNA/protein level may be more valuable in predicating prognosis. As reported previously, FBXW7 mutated CRC patients resistant to anti-epidermal growth factor receptor (EGFR) immunotherapy treatment (monoclonal antibodies, Cetuximab or Panitumumab) [30]. Besides, loss of FBXW7 is associated with drug resistance to Oxaliplatin [31]. It has been reported that rapamycin could inhibit FBXW7 loss-induced epithelialmesenchymal transition and cancer stem cell-like characteristics in CRC cells $[6,26]$. And rapamycin could inhibit tumor metastasis in vivo in cholangiocarcinoma [27]. However, application of FBXW7 signaling pathway targeted therapies in human is no clue yet.

This meta-analysis has several limitations to be discussed. First, significant heterogeneity was observed among the included studies. By excluding each study individually, sensitivity analysis revealed that the predictive significance of FBXW7 status on OS in CRC. Second, there was some unavoidable variability in study designs, such as the sequence method or antibody used for
FBXW7 mutation or expression detection, TNM stage of the involved patients and the cutoff value for dichotomizing FBXW7 low or high expression. And the variability among studies is indeed a problem when determining the significance of FBXW7. Fortunately, publication bias was not detected for all the studies for OS and four studies for DFS, and sensitivity analysis revealed that no significant change was found in the results when any 1 study was excluded. Third, several studies having small numbers of patients recruited. Finally, publication bias may be a problem in metaanalyses though not detected using Begg's and Egger's tests. All relevant data were tired to identified, and additional unpublished information was retrieved, but some missing data were unavoidable.

\section{Conclusions}

Altered FBXW7 status was associated with advanced $\mathrm{T}$ stage and lymph node metastasis in CRC, and low FBXW7 mRNA/protein level indicates poor OS in CRC. FBXW7 may be a potential prognostic biomarker in CRC patients. These findings may provide evidence for determining therapeutic regimen in CRC patients.

\section{Acknowledgements \\ Not applicable.}

\section{Authors' contributions}

$\mathrm{HY}$ and JBC come up with the study, then all author collaborated on the design of the project. WS, WCY and RL collated, screened and analyzed the data together, and drafted the manuscript. LLC, DDC, LH and WGY reviewed the content, revised the manuscript and approved the final manuscript. With the joint efforts of all authors, this study was completed and completed. The author(s) read and approved the final manuscript.

Funding

No funding was obtained for this study. 


\section{Availability of data and materials}

The datasets used and/or analyzed during the current study are available from the corresponding author on reasonable request.

\section{Declarations}

Ethics approval and consent to participate

Not applicable.

\section{Consent for publication}

Not applicable.

\section{Competing interests}

Nothing to declare.

\section{Author details}

'Department of General Surgery, Key Laboratory of Metabolism and Gastrointestinal Tumor, Key Laboratory of Laparoscopic Technology, Shandong Medicine and Health Key Laboratory of General Surgery, The First Affiliated Hospital of Shandong First Medical University \& Shandong Provincial Qianfoshan Hospital, the First Affiliated Hospital of Shandong First Medical University, Jinan 250000, Shandong, China. ${ }^{2}$ Department of General Surgery, Shandong Provincial Qianfoshan Hospital, Weifang Medical College, Weifang 261000, Shandong, China. ${ }^{3}$ Department of Pathology, Jinan Central Hospital, Jinan 250000, Shandong, China. ${ }^{4}$ Department of General Surgery, Feicheng Hospital of Shandong Guoxin Yiyang Group, Tai'an 271600, Shandong, China. ${ }^{5}$ Department of Gastrointestinal Surgery, Zibo First People's Hospital, Zibo 255000, Shandong, China.

Received: 15 December 2020 Accepted: 23 June 2021 Published online: 03 July 2021

\section{References}

1. Bray F, Ferlay J, Soerjomataram I, Siegel RL, Torre LA, Jemal A. Global Cancer statistics 2018: GLOBOCAN estimates of incidence and mortality worldwide for 36 cancers in 185 countries. CA Cancer J Clin. 2018;0(0):1-31.

2. Siegel RL, Miller KD, Goding Sauer A, Fedewa SA, Butterly LF, Anderson JC, et al. Colorectal cancer statistics, 2020. CA Cancer J Clin. 2020;70(3):145-64. https://doi.org/10.3322/caac.21601.

3. Keum N, Giovannucci E. Global burden of colorectal cancer: emerging trends, risk factors and prevention strategies. Nat Rev Gastroenterol Hepatol. 2019;16(12):713-32. https://doi.org/10.1038/s41575-019-0189-8.

4. Xue L, Williamson A, Gaines S, Andolfi C, Paul-Olson T, Neerukonda A, et al. An update on colorectal cancer. Curr Probl Surg. 2018;55(3):76-116. https:// doi.org/10.1067/j.cpsurg.2018.02.003.

5. Welcker M, Clurman BE. FBW7 ubiquitin ligase: a tumour suppressor at the crossroads of cell division, growth and differentiation. Nat Rev Cancer. 2008; 8(2):83-93. https://doi.org/10.1038/nrc2290.

6. Yumimoto K, Nakayama Kl. Recent insight into the role of FBXW7 as a tumor suppressor. Semin Cancer Biol. 2020;67(Pt 2):1-15. https://doi.org/10.1 016/j.semcancer.2020.02.017.

7. Akhoondi S, Sun D, von der Lehr N, Apostolidou S, Klotz K, Maljukova A, et al. FBXW7/hCDC4 is a general tumor suppressor in human cancer. Cancer Res. 2007;67(19):9006-12. https://doi.org/10.1158/0008-5472.CAN-07-1320.

8. Tan Y, Sangfelt O, Spruck C. The Fbxw7/hCdc4 tumor suppressor in human cancer. Cancer Lett. 2008;271(1):1-12. https://doi.org/10.1016/j.canlet.2008.04.036.

9. Yeh $\mathrm{CH}$, Bellon M, Nicot C. FBXW7: a critical tumor suppressor of human cancers. Mol Cancer. 2018;17(1):115. https://doi.org/10.1186/s12943-018-0857-2.

10. Karczmarski J, Goryca K, Pachlewski J, Dabrowska M, Pysniak K, Paziewska A, et al. Mutation profiling of premalignant colorectal neoplasia. Gastroenterol Res Pract. 2019;2019:2542640.

11. Chang C-C, Lin H-H, Lin J-K, Lin C-C, Lan Y-T, Wang H-S, et al. FBXW7 mutation analysis and its correlation with clinicopathological features and prognosis in colorectal cancer patients. Int J Biol Markers. 2015;30(1):E88-95. https://doi.org/10.5301/jbm.5000125.

12. Gao XH, Yu GY, Hong YG, Lian W, Chouhan H, Xu Y, et al. Clinical significance of multiple gene detection with a 22-gene panel in formalinfixed paraffin-embedded specimens of 207 colorectal cancer patients. Int $J$ Clin Oncol. 2019;24(2):141-52. https://doi.org/10.1007/s10147-018-1377-1.
13. He D, Ma Z, Fang C, Ding J, Yang W, Chen P, et al. Pseudophosphatase STYX promotes tumor growth and metastasis by inhibiting FBXW7 function in colorectal cancer. Cancer Lett. 2019;454:53-65. https://doi.org/10.1016/j.canlet.2019.04.014.

14. Iwatsuki M, Mimori K, Ishii H, Yokobori T, Takatsuno Y, Sato T, et al. Loss of FBXW7, a cell cycle regulating gene, in colorectal cancer: clinical significance. Int J Cancer. 2010;126(8):1828-37. https://doi.org/10.1002/ijc.24879.

15. Kawashita Y, Morine Y, Ikemoto T, Saito Y, Iwahashi S, Yamada S, et al. Loss of Fbxw7 expression is a predictor of recurrence in colorectal liver metastasis. J Hepatobiliary Pancreat Sci. 2017;24(10):576-83. https://doi.org/10.1002/jhbp.500.

16. Korphaisarn K, Morris VK, Overman MJ, Fogelman DR, Kee BK, Raghav KPS, et al. FBXW7 missense mutation: a novel negative prognostic factor in metastatic colorectal adenocarcinoma. Oncotarget. 2017;8(24):39268-79. https://doi.org/10.18632/oncotarget.16848.

17. Li Q, Li Y, Li J, Ma Y, Dai W, Mo S, et al. FBW7 suppresses metastasis of colorectal cancer by inhibiting HIF1a/CEACAM5 functional axis. Int J Biol Sci. 2018;14(7):726-35. https://doi.org/10.7150/ijbs.24505.

18. Liu H, Wang K, Fu H, Song J. Low expression of the ubiquitin ligase FBXW7 correlates with poor prognosis of patients with colorectal cancer. Int J Clin Exp Pathol. 2018;11(1):413-9.

19. Mouradov D, Domingo E, Gibbs P, Jorissen RN, Li S, Soo PY, et al. Survival in stage II/III colorectal cancer is independently predicted by chromosomal and microsatellite instability, but not by specific driver mutations. Am J Gastroenterol. 2013;108(11):1785-93. https://doi.org/10.1038/ajg.2013.292.

20. Vicente Cavagnari MA, Silva TD, Haddad Pereira MA, Sauer $\sqcup$, Shigueoka D, Saad SS, et al. Impact of genetic mutations and nutritional status on the survival of patients with colorectal cancer. BMC Cancer. 2019;19. https:// www.ncbi.nlm.nih.gov/pmc/articles/PMC6599287/.

21. Parmar MK, Torri V, Stewart L. Extracting summary statistics to perform meta-analyses of the published literature for survival endpoints. Stat Med. 1998;17(24):2815-34. https://doi.org/10.1002/(SICI)1097-0258(19981230)17:24 $<2815:$ :AID-SIM110>3.0.CO;2-8.

22. Williamson PR, Smith CT, Hutton JL, Marson AG. Aggregate data metaanalysis with time-to-event outcomes. Stat Med. 2002;21(22):3337-51. https://doi.org/10.1002/sim.1303.

23. Tierney JF, Stewart LA, Ghersi D, Burdett S, Sydes MR. Practical methods for incorporating summary time-to-event data into meta-analysis. Trials. 2007;8(1). https://pm.yuntsg.com/details.html?pmid=17555582\&key=17555582.

24. Tang H, Zhao T, Sang W. Clinical significance of FBXW7 and c-myc protein expression in colorectal cancer. Anhui Medical Journal. 2016;36(6):705-7.

25. Zhan $P$, Wang $Y$, Zhao S, Liu C, Wang $Y$, Wen $M$, et al. FBXW7 negatively regulates ENO1 expression and function in colorectal cancer. Lab Investig. 2015;95(9):995-1004. https://doi.org/10.1038/labinvest.2015.71.

26. Wang Y, Liu Y, Lu J, Zhang P, Wang Y, Xu Y, et al. Rapamycin inhibits FBXW7 loss-induced epithelial-mesenchymal transition and cancer stem cell-like characteristics in colorectal cancer cells. Biochem Biophys Res Commun. 2013;434(2):352-6. https://doi.org/10.1016/j.bbrc.2013.03.077.

27. Yang H, Lu X, Liu Z, Chen L, Xu Y, Wang Y, et al. FBXW7 suppresses epithelialmesenchymal transition, stemness and metastatic potential of cholangiocarcinoma cells. Oncotarget. 2015;6(8):6310-25. https:/doi.org/10.18632/oncotarget.3355.

28. Wang $Y$, Zhang $P$, Wang $Y$, Zhan $P$, Liu C, Mao J-H, et al. Distinct interactions of EBP1 isoforms with FBXW7 elicits different functions in cancer. Cancer Res. 2017;77(8):1983-96. https://doi.org/10.1158/0008-5472.CAN-16-2246.

29. Kemp Z, Rowan A, Chambers W, Wortham N, Halford S, Sieber O, et al. CDC4 mutations occur in a subset of colorectal cancers but are not predicted to cause loss of function and are not associated with chromosomal instability. Cancer Res. 2005;65(24):11361-6. https://doi.org/1 0.1158/0008-5472.CAN-05-2565.

30. Lupini L, Bassi C, Mlcochova J, Musa G, Russo M, Vychytilova-Faltejskova P, et al. Prediction of response to anti-EGFR antibody-based therapies by multigene sequencing in colorectal cancer patients. BMC Cancer. 2015;15(1): 808. https://doi.org/10.1186/s12885-015-1752-5.

31. Li N, Lorenzi F, Kalakouti E, Normatova M, Babaei-Jadidi R, Tomlinson I, et al. FBXW7-mutated colorectal cancer cells exhibit aberrant expression of phosphorylated-p53 at Serine-15. Oncotarget. 2015;6(11):9240-56. https:// doi.org/10.18632/oncotarget.3284.

\section{Publisher's Note}

Springer Nature remains neutral with regard to jurisdictional claims in published maps and institutional affiliations. 